\title{
UMA COMPREENSÃO DOS FUNDOS ARQUIVÍSTICOS SOB O ENFOQUE DA ANÁLISE DE DOMÍNIO
}

\section{LA COMPRENSIÓN DE LOS FONDOS ARCHIVÍSTICOS MEDIANTE EL ENFOQUE DEL ANÁLISIS DE CAMPO}

\begin{abstract}
Leandro Ribeiro Negreiros - leandro negreiros@hotmail.com
Mestre em Ciência da Informação pelo Programa de Pós-Graduação da Escola de Ciência da Informação da Universidade Federal de Minas Gerais (UFMG). Co-coordenador e Professor convidado do curso de especialização Gestão de Arquivos e Documentos da Pontifícia Universidade Católica de Minas Gerais.
\end{abstract}

\begin{abstract}
Resumo
O trabalho procura buscar uma aproximação com a teoria de Birger Hjørland, identificando as instituições públicas e privadas produtoras de documentos arquivísticos como domínios específicos de conhecimento, que: (a) produzam documentos decorrentes de suas atividades e funções; (b) possuam terminologia especializada; (c) requeiram análises estruturais e históricas para a determinação da organicidade dos documentos e estabelecimento do princípio de proveniência; (d) abranjam uma comunidade de usuários com necessidades de acesso a documentos e necessidades informacionais complexas e específicas; e (e) utilizam sistemas inteligentes para recuperação e organização de documentos arquivísticos. Utilizando-se as abordagens propostas será possível alcançar melhorias na gestão de documentos arquivísticos no que se refere a manutenção de proveniência e autenticidade dos documentos, otimização de sistemas informatizados de gerenciamento de documentos e eficácia na recuperação da informação em arquivos.
\end{abstract}

Palavras-chave: Análise de Domínio; Gestão de Documentos Arquivísticos; Organização da Informação. Recuperação da Informação. Arquivos. 


\section{INTRODUÇÃO}

Grande parte das propostas de implementação de sistemas informatizados de gerenciamento de documentos em arquivos inicia-se com o acúmulo de documentos, com a desorganização, com a inacessibilidade aos documentos arquivísticos e com os problemas jurídicos e administrativos que derivam dessa realidade. Propostas que prometem solucionar em curto prazo esse complexo cenário, tendem a supervalorizar as tecnologias e negligenciar a enorme estrutura que produziu, recebeu, utilizou e necessita, ainda, utilizar os documentos oriundos das atividades e funções das instituições. Da maneira como o processo é realizado três situações são previstas:

Primeiro, que a desordem aparente se transfira para um ambiente eletrônico, em que os sistemas informatizados de gerenciamento de documentos arquivísticos tornam-se superlotados de dados, dando a falsa impressão à comunidade de usuários que o utiliza de que tanto os documentos quanto seus conteúdos estão disponíveis. No entanto, a inconsistência e a falta de padrões para inserção dos dados não permitem que os resultados esperados - o respeito pela proveniência, a eficiência da recuperação, o fácil acesso e a garantia dos documentos como elementos de prova e testemunho sejam alcançados.

Segundo, que os documentos

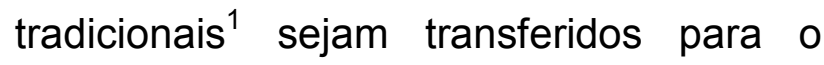
ambiente eletrônico - através de digitalização, por exemplo - e seus originais destruídos logo em seguida, na tentativa de reduzir custos com a documentação e a liberação de espaços. Nesses casos, a supervalorização dos sistemas informatizados de gerenciamento de documentos arquivísticos é ainda maior, e problemas como obsolescência tecnológica, integridade e fidedignidade dos documentos e o princípio de proveniência, são deixados de lado, negligenciando a organicidade dos documentos, a representação da instituição que os produziu e os valores de prova e testemunho dos documentos.

Terceiro que, instituições que aplicam soluções imediatistas de sistemas informatizados de gerenciamento de documentos acreditem que estão realizando gestão de informação, quando, na maioria das vezes, sequer organizaram efetivamente seus documentos arquivísticos. A gestão de informações para tomada de decisão só é possível

\footnotetext{
${ }^{1}$ Entende-se por tradicionais aqueles documentos produzidos, comumente, em papel.
} 
quando há a gestão de documentos arquivísticos e não arquivísticos.

Partindo dessa realidade, é preciso que se determine, através da teoria, maneiras de estudar 0 contexto organizacional em que os documentos arquivísticos originam, para que a produção, uso e disseminação dos mesmos possam, efetivamente, ocorrer. Com base nessa premissa, procurou-se na abordagem social de Birger Hjørland pontos comuns com a teoria e a prática arquivísticas que pudessem ser detectados, metodologias que permitissem ser aplicadas e reflexões que propusessem intercâmbio entre as áreas de Ciência da Informação e Arquivologia.

Nesse sentido, o objetivo do presente artigo é buscar uma aproximação com a teoria de Hjørland, esforçando-se por identificar as instituições públicas e privadas produtoras de documentos arquivísticos como domínios específicos, que produzem documentos decorrentes de suas atividades e funções; possuem terminologia especializada; requerem análises estruturais e históricas para a determinação da organicidade dos documentos e estabelecimento do princípio de proveniência; abrangem uma comunidade de usuários com necessidades de acesso a documentos e necessidades informacionais complexas e específicas; e utilizam sistemas inteligentes para recuperação e organização de documentos arquivísticos. Não se trata, entretanto, de analisar e mapear o conhecimento da arquivologia, mas de considerar a instituição produtora $\mathrm{e}$ receptora dos documentos arquivísticos como o ambiente, ou seja, um domínio específico a ser estudado profundamente.

\section{A ANÁLISE DE DOMÍNIO}

Para Hjørland (2004, p. 4), um domínio "pode ser uma disciplina, um campo escolar. Pode ser ainda uma comunidade discursiva conectada a um partido político, à religião, ao comércio, ou a um lazer". Ele relaciona esses domínios específicos às práticas da Ciência da Informação, mostrando o que o domínio pode realizar para melhorar as práticas informacionais, prover melhor os serviços de informação e integrar diversos campos de pesquisa, como a bibliometria e a recuperação da informação.

Trata-se de uma abordagem socioepistemológica, funcionalista e realista, que procura localizar as bases da Ciência da Informação externamente às percepções individualistas e subjetivas dos usuários, em oposição à abordagem cognitivista. $O$ conceito de informação, nesse sentido, só 
tem importância em relação a um pressuposto conhecido e compartilhado entre os diversos membros da comunidade. A análise de domínio adota uma concepção sócio-cognitiva, em que são valorizados os papéis da cultura e sociedade na cognição. Em detrimento das representações simbólicas, as pesquisas em semântica e pragmática são enfatizadas.

Hjørland (2002) defende que os recursos informacionais devem ser tratados, ou seja, identificados, descritos, organizados e disseminados para servirem a um objetivo específico. Isto corrobora com a idéia de que, em arquivos, prima-se pela organização dos documentos para uma posterior utilização da informação, tendo como base o contexto da instituição que os produziu e aos objetivos, funções e atividades que os originaram. Muito embora os objetivos de documentos arquivísticos sirvam determinantemente como provas de ações, isso não os redime de serem insumos para a tomada de decisão das instituições e de servirem à pesquisa e a história.

O autor apresenta onze abordagens para a análise de domínio:

1. Produção de guias de literatura (Producing literature guides or subject gateways);
2. Construção de classificações e tesauros especializados (Constructing special classifications and thesauri);

3. Indexação e recuperação da informação especializada (Indexing and retrieving spe-cialities);

4. Estudos empíricos de usuários (Empirical user studies);

5. Estudos bibliométricos (Bibliometrical studies);

6. Estudos históricos (Historical studies);

7. Estudos de documentos e gêneros (Document and genre studies);

8. Estudos epistemológicos e críticos (Epistemological and critical studies);

9. Estudos terminológicos, línguagens para propósitos determinados, semântica de bases de dados e estudo dos discursos (Terminological studies, language for special purpose - LSP, database semantics and discourse studies);

10. Estrutura e instituições da comunicação científica (Structures and institutions in scientific communication);

11. Cognição científica, conhecimento perito e inteligência artificial 
(Scientific cognition, expert kowledge and artificial intelligece $-A l)$.

As abordagens em Hjørland (1998, 2002, 2004) estão relacionadas principalmente à análise de domínios específicos; a maioria de suas aplicações (ZINS; GUTTMANN, 2003, é um exemplo) utilizam a metodologia para o estudo e entendimento de campos específicos do conhecimento. Sendo assim, a instituição na qual o arquivo e seu conjunto de documentos está inserido será como um domínio a ser analisado para que o processo de organização e recuperação possa ser realizado de acordo com as necessidades específicas desse domínio e a solução de sistema informatizado de gerenciamento de documentos, siga o mesmo caminho. O exercício que se pretende realizar será relacionar cada uma das abordagens de Hjørland com as práticas arquivísticas.

\section{AS ABORDAGENS E SUA RELAÇÃO COM A ARQUIVOLOGIA}

As origens já explicitadas dos estudos de Hjørland serão determinantes para que algumas abordagens não se enquadrem na análise que ora se realiza. Estas abordagens, no entanto, serão apresentadas no final desse tópico. Nesta seção, a aproxi- mação da teoria do autor pode ser assim explicitada:

a) Produção de guias de literatura (Producing literature guides or subject gateways):

Hjørland (2002) define guias como publicações que listam e descrevem sistemas de fontes de informações em uma ou mais áreas. É um tipo de bibliografia de documentos de um domínio. A metodologia, nessa abordagem, consistiria em procedimentos que selecionariam os documentos em um domínio específico; classificaria de acordo com regras determinadas relacionadas com o contexto; descreveria as características das referências; selecionaria as mais importantes e proveria guias de como acessar as fontes de informação.

Em arquivos permanentes é fundamental a produção de instrumentos de recuperação para que o acervo torne-se conhecido e seu acesso seja facilitado. Os procedimentos metodológicos utilizados na elaboração de instrumentos de recuperação arquivísticos são os mesmos listados por Hjørland, com a diferença de não se tratar de fontes bibliográficas e, por isso, constituírem documentos únicos, inexistentes em outras instituições. Enquanto que a Ciência da Informação se incumbiria de produzir guias que relacionassem a produção bibliográfica de uma determinada área, cabe a arquivologia produzir guias e de- 
mais instrumentos de recuperação relacionados a instituições ou a instituições arquivísticas em específico. Um grande problema é a rápida obsolescência, o que poderia ser solucionado com a produção desses instrumentos em ambiente eletrônico.

Cada uma das tipologias de instrumentos de recuperação em arquivos servem a um objetivo específico. Segundo Paes (2005, p. 127-139, grifo nosso), os tipos básicos de instrumentos de pesquisa em arquivos são:

Guia: Obra destinada à orientação dos usuários no conhecimento e na utilização dos fundos que integram - acervo de um arquivo permanente. É $O$ instrumento de pesquisa mais genérico, pois se propõe a informar sobre a totalidade dos fundos existentes no arquivo; [...]

Inventário: $O$ inventário sumário é o instrumento no qual as unidades de arquivamento de um fundo ou de uma de suas divisões são identificadas e descritas sucintamente. Sua finalidade é descrever a composição do fundo e ao mesmo tempo promover o arquivo de um instrumento preliminar de busca para cada fundo. $O$ inventário analítico é o instrumento de pesquisa no qual as unidades de arquivamento de um fundo, ou de uma de suas divisões são identificas e descritas pormenorizadamente; [...]

Catálogo: Instrumento de pesquisa elaborado segundo um critério temático, cronológico, onomástico ou geográfico, incluindo todos os documentos, pertencentes a um ou mais fundos, descritos de forma sumária ou pormenorizada; [...]

Repertório: É um instrumento de pesquisa que descreve

\begin{abstract}
pormenorizadamente documentos previamente selecionados, pertencentes a um ou mais fundos, segundo um critério temático, cronológico, onomástico ou geográfico; $[\ldots]$

Îndice: É uma lista sistemática, pormenorizada, dos elementos do conteúdo de um documento ou grupo de documentos, disposta em determinada ordem para indicar sua localização no texto. Sua finalidade é remeter o leitor onde se acha inserido o termo indexado e apresenta-se em duas formas: como obra independente ou como parte integrante da obra indexada [...].
\end{abstract}

Com o surgimento das tecnologias da informação, a ênfase nos documentos arquivísticos muda, recaindo sobre os próprios sistemas informatizados de gerenciamento arquivístico de documentos. Dollar (1990 apud Jardim, 1992) argumenta sobre a necessidade de se preocupar com a configuração dos sistemas e Jardim (1992, p. 255) corrobora com essa idéia mostrando que é "essencial a implementação de um sistema Diretório sobre Recursos de Gestão da Informação (bases de dados sobre outras bases de dados)". O Arquivo Nacional, por exemplo, possui, em seu site, diversos exemplos em que é possível consultar acervos relacionados a temas específicos através de uma única base de da$\operatorname{dos}^{2}$.

2 O Centro de Informação de Acervos dos Presidentes da República (2007) possui uma base de dados que serve de instrumento de localização e reconstituição dos acervos dos presidentes da república. "Chamado à épo- 
Sistemas informatizados de gerenciamento arquivístico de documentos, se parametrizados e customizados adequadamente, podem atender às necessidades específicas de levantamento, criação de guias, inventários etc., tornando esses instrumentos menos suscetíveis a obsolescência. A gestão de documentos arquivísticos estaria tão bem servida pelos relatórios que podem ser configurados e emitidos pelos sistemas, referentes às estatísticas e ao conteúdo da documentação nele disposta.

b) Construção de classificações e tesauros especializados (Constructing special classifications and thesauri):

Hjørland (2002, p. 425) aponta que a literatura sobre classificação de domínios específicos são limitadas em quantidade e metodologia. A maioria das pesquisas em classificação estão direcionadas para os esquemas de classificação universais (como por exemplo, a Classificação Decimal de Dewey e a Classificação Decimal Universal, ambas bibliográficas) e pouco tem sido feito para o desenvolvimento de propostas para áreas específicas.

ca Centro de Referência de Acervos Privados Presidenciais, seus fundamentos foram estabelecidos a partir da reunião de diferentes instituições que cuidaram da elaboração da metodologia e estruturação dos dados, das coletas específicas e de sua manutenção e outras tantas ações".
Em arquivos a realidade é outra, mas o problema se apresenta da mesma maneira. As classificações bibliográficas, assim como as classificações científicas, diferem das classificações arquivísticas. A classificação em arquivos refere-se à organização dos documentos de acordo com o quadro de arranjo pré-estabelecido. Este quadro de arranjo está intimamente ligado à organização que produziu os documentos. Por isso não se pode falar em classificação universal para arquivos. De qualquer forma classificações por assunto $^{3}$ não são costumeiramente aplicáveis a conjuntos de documentos e os planos de classificações em arquivos são desenvolvidos, sobremaneira, tendo por base as funções e atividades nas quais originaram os registros documentais.

O termo classificação em arquivos assume ainda outras acepções, como por exemplo, o de atribuir graus de sigilo aos documentos segundo as informações que esses contêm. De qualquer maneira, essa classificação de segurança será determinada também de acordo com as exigências

\footnotetext{
${ }^{3}$ Práticas de classificação por assunto foram muito comuns durante o período iluminista, na Europa, principalmente pelas localidades que consideravam uma proveniência única para todos os arquivos do Estado. França e Rússia, são exemplos de países que trataram seus conjuntos documentais dessa maneira, também denominada como método inorgânico. As instruções de 24 de abril de 1841, feitas por Natalis de Wailly, anunciaram o princípio do respeito pelos fundos - funda-
} 
do domínio, ou seja, da proveniência em que se inserem os documentos.

c) Indexação e recuperação da informação especializada (Indexing and retrieving specialities):

$\mathrm{O}$ autor afirma que a indexação e a recuperação da informação são sempre específicas. Os estudos da Ciência da Informação têm, segundo Hjørland (2002), ignorado o modo como domínios diversos demandam os diferentes sistemas para organizar e recuperar documentos. $O$ autor ainda defende que focar mais fortemente as necessidades de domínios diferentes pode tornar os estudos mais realistas e os profissionais mais relevantes.

Com o contexto arquivístico o mesmo ocorre. Apesar da indexação estar, freqüentemente, voltada para arquivos permanentes, em que o foco das pesquisas é informacional e/ou histórica, nada impede que se utilize a extração de termos dos documentos em arquivos correntes para a realização da gestão de documentos e de comodidade para a localização e acesso aos mesmos. Pensar a recuperação da informação de acordo com o contexto de produção dos documentos e a organização que os originou, resguardará, sobretudo, o valor do princípio de proveniência. Diver-

mento essencial da arquivística em contraposição à ordenação por assuntos (SILVA et al., 1999). sas iniciativas (EUROPEAN COMISSION INTERCHANGE OF DATA BETWEEN ADMINISTRATIONS - IDA, 2001; CONSELHO NACIONAL DE ARQUIVOS - CONARQ, 2006, são exemplos) têm definido conjuntos de metadados para documentos arquivísticos, que ajudariam na descrição e facilitariam a recuperação.

d) Estudos empíricos de usuários (Empirical user studies):

A maioria dos estudos de usuários desenvolvidos em unidades de informação espera que os sujeitos reajam de uma maneira mecânica e homogênea em diferentes situações, sem considerar as determinações culturais do ambiente que atuam e sem considerar os objetivos e os valores dos conteúdos e dos documentos. Hjørland (2002) afirma que é preciso levar em conta o contexto e a tarefa. Muitas vezes os usuários não sabem quais documentos procurar e, quando estes são localizados, não conseguem determinar quais informações precisam. Conseqüentemente, os cientistas de informação não podem esperar aprender como organizar e procurar a informação a partir de estudos de usuários.

O mesmo ocorrerá com a arquivologia. Os estudos de usuários devem partir da organização do fundo, ou seja, entender primeiro o método e a origem de desenvolvimento do conjunto de documentos arquivísticos para, a posteriori, tentar entender a 
maneira pela qual os documentos são buscados e utilizados pelos usuários do arquivo. Isto porque além do usuário de arquivos ter dúvidas quanto ao que buscar e quanto à informação que necessita, suas necessidades podem ser pré-determinadas pela fase do ciclo de vida dos documentos. Sendo assim, espera-se que o usuário de arquivos correntes necessite de documentos arquivísticos indispensáveis à manutenção das atividades cotidianas de uma administração. Tais documentos serão de uso freqüente e, por isso, devem permanecer o mais perto possível do utilizador ou, se constituírem documentos eletrônicos, serem facilmente acessíveis. Ao contrário, os usuários de arquivos permanentes, geralmente pesquisadores, não se importam de deslocar-se até uma instituição onde estão custodiados os documentos não mais de valor probatório e administrativo, mas de valor histórico-informacional. Observase, assim, que a fase em que se encontra o documento em arquivos é que determinará o tipo de usuário que dele terá acesso e as próprias condições de acesso (sigilo e segurança).
e) Estudos históricos (Historical stu- dies):

Hjørland (2002) conclui que não se deve considerar somente os estudos históricos como uma maneira de configurar um campo como uma área mais nobre. Os mé- todos históricos devem ser considerados como substanciais na Ciência da Informação. Quando relacionados aos documentos, às organizações, aos sistemas, ao conhecimento e à informação, a perspectiva histórica, bem como os métodos históricos podem representar estudos mais profundos e coerentes.

Nos fundos arquivísticos a história do fundo e a história administrativa de seu responsável são determinantes para o bom entendimento do processo de produção dos documentos, do contexto de uso dos mesmos, ou seja, da compreensão da proveniência dos documentos. A perspectiva histórica do domínio arquivístico é essencial para a elaboração da classificação, para a determinação do modo de descrição, inclusive do vocabulário a ser adotada na indexação.

f) Estudos de documentos e gêneros (Document and genre studies):

Hjørland (2002) pressupõe que os conceitos de arquitetura da informação, estruturação de informação, introdução de sistemas de recuperação de textos completos e sistemas de recuperação da informação baseados em linguagens de hipertexto e HTML, por exemplo, devem ser relacionados a teorias mais gerais dos documentos, suas finalidades e funções comunicativas, seus elementos e composições e seus valores potenciais na recuperação de in- 
formação. A diversidade de tipos de documentos pode ser examinada de acordo com as ações comunicativas ou de acordo com as finalidades a que os documentos foram produzidos. Mas, entretanto, estudos quantitativos e qualitativos mais aprofundados das tipologias documentais em comunidades diferentes podem fornecer serviços de informação mais ricos e diferenciados. A análise de gênero dos documentos é inspiradora para o desenvolvimento de novas metodologias e enfatiza as dimensões sociais e históricas da comunicação.

Nos arquivos, antes de se conceber a implementação de um sistema informatizado de gerenciamento de documentos e para se entender as relações informacionais existentes nas instituições, é preciso determinar os gêneros e a espécies e os tipos e categoria de documentos produzidos pela instituição que acumulou o acervo.

Tais estudos podem definir, por exemplo, a necessidade de organização dos documentos, o nível de descrição, a complexidade da classificação, as exigências da indexação e, sobretudo, as relações orgânicas dos documentos produzidos pela entidade. Para a produção de documentos, a análise tipológica pode gerar diretrizes para a racionalização da produção e meIhoramento do fluxo de documentos na instituição. Com relação ao uso, é possível melhor a padronização do documento e de seu conteúdo, facilitando-se assim o seu entendimento, acesso e disseminação. A avaliação, a partir da análise dos documentos, passa a ser mais criteriosa, pois os prazos de guarda podem ser definidos quando da avaliação da tipologia documental e a destinação pode ser mais adequada do que somente a baseada em legislação.

g) Estudos terminológicos, linguagens para propósitos determinados, semântica de bases de dados e estudo dos discursos (Terminological studies, language for special purpose (LSP), database semantics and discourse studies):

Profissionais da informação, afirma Hjørland (2002), sempre tiveram problemas relacionados à terminologia, semântica ou outros problemas de natureza lingüística: a construção de tesauros, problemas relacionados à eficiência de busca por linguagem natural ou controlada etc. O autor afirma que esta problemática deveria ser orientada para os estudos de lingüistas, que tendem a ser relutantes em considerar "sublinguagens".

Para Hjørland (2002), sublinguagens, ou linguagens para propósitos determinados (Language for Special Purpose - LSP), são parcelas da linguagem natural que diferem sintaticamente e/ou lexicalmente de outras parcelas da mesma linguagem: 
[Uma sublinguagem é] a linguagem particular que foi usada no corpo dos textos que tratam de uma área específica (freqüentemente relatórios ou artigos de um subcampo técnico de uma especialidade ou da ciência), em que os autores dos documentos compartilham de um vocabulário comum e de hábitos comuns do uso da palavra e da escrita (HIRSCHMAN; SAGER, 1982, p. 28, apud HJØRLAND, 2002, p. 443, tradução nossa).

Os princípios fundamentais da LSP são determinados de um lado pelas necessidades de comunicação de diferentes grupos e, por outro, pelos princípios econômicos, que reduzem a redundância da informação. Hjørland acredita que a aproximação da LSP e a semântica de base de dados na Ciência de Informação estão distante de estudos de natureza teórica e estão conectadas a quatro suposições principais:

1 Símbolos (signs) e seus significados são formados por grupos sociais como parte da divisão social do trabalho na sociedade;

2 Diferentes comunidades desenvolvem tipos específicos de documentos. Todos os elementos nesses documentos são potencialmente pontos de acesso na recuperação eletrônica. $\mathrm{O}$ valor da informação de um ponto de acesso em específico está relacionado às convenções usadas em um domínio específico;

3 As comunidades discursivas são influenciadas sempre pelas várias normas e tendências epistemológicas, que influenciam também a construção social de sistemas simbólicos, de mídia, de conhecimento, de significado e de distâncias semânticas;

4 Quando os documentos são inseridos em bases de dados informacionais a partir de significados implícitos em contextos prévios ele ficarão perdidos. Os sistemas de organização do conhecimento e de recuperação de informação devem ser desenvolvidos para lidar com esta perda de informação implícita preocupando-se com a explícita, ou seja, com s semântica da base de dados.

$O$ estudo de linguagens e o estudo de terminologias são muito importantes para a Ciência da Informação porque o uso efetivo desses ferramentais influenciará no modo de pensar e na proposição de termos de pesquisa em bases de dados pelos integrantes de um domínio.

Em arquivos a importância da linguagem não muda. $O$ fato dos documentos arquivísticos surgirem a partir das atividades administrativas, funcionais e legais das ins- 
tituições, é inerente afirmar que esses documentos refletirão a linguagem própria de seus produtores. Sendo assim, para se determinar a linguagem específica que rege a produção dos documentos arquivísticos de uma instituição, cabe um profundo estudo sobre a proveniência desses documentos. $O$ entendimento do que representa o arquivo para uma determinada instituição; o nível de padronização da produção dos documentos; os meios efetivos de comunicação entre os integrantes da mesma; e os significados que estes atribuem aos termos empregados na produção dos documentos são cruciais para a concepção de um vocabulário controlado que reflita as necessidades informacionais de seus usuários.

h) Cognição científica, conhecimento perito e inteligência artificial - IA (Scientific cognition, expert kowledge and artificial intelligence $A l)$ :

O autor aponta as ligações da proposta de análise de domínio com a Ciência da Computação. "A análise do domínio é um método usado no desenvolvimento dos sistemas e na tecnologia de programação" (HJØRLAND, 2002, p. 449). O ponto de vista básico é que um sistema ou um serviço podem refletir um domínio, mas na Ciência da Informação, entretanto, não se considera somente o desenvolvimento de sistemas para computador, mas se tem a necessidade de alargar e compreender disciplinas e grupos de usuários diferentes, com perspectivas sociológicas ou humanísticas.

Tanto a IA e as Ciências Cognitivas estão relacionadas historicamente por maneiras individualistas de pensar do que sociais. Há muitas pesquisas sobre como investigadores e peritos compreendem domínios diferentes do conhecimento. Algumas delas estão relacionadas à tentativa de construir os sistemas peritos com inteligência artificial. Tais pesquisas são realizadas, em grande parte, sob um ponto de vista mecânico do pensar humano, negligenciando os aspectos históricos e culturais da cognição humana. (HJØRLAND, 2002).

O autor ressalta que os objetivos da Ciência da Informação não são os mesmos da Inteligência Artificial. Na Ciência da Informação os objetivos centrais são fornecer aos usuários informações que possam validar diferentes reivindicações. "Isto é muito diferente de construir Inteligência Artificial" (HJØRLAND, 2002, p. 450).

Pode-se afirmar também que os objetivos da Arquivologia não são os mesmos da Inteligência Artificial. Em arquivos, a princípio, o objetivo maior é fornecer o acesso aos documentos, guardadas as restrições e os valores informacionais. Uma vez realizada a gerência de documentos, 
pode-se realizar aquilo que a literatura denomina de gestão da informação. $O$ desenvolvimento de sistemas eletrônicos para solucionar os problemas de acessibilidade e organização de documentos em instituições que produzem grandes quantidades documentais não deve também negligenciar os aspectos históricos e culturais que abarcam uma instituição que vem se desenvolvendo ao longo de anos. O sistema desenvolvido deverá ser capaz de determinar a localização dos documentos; assegurar a distribuição dos mesmos; torná-los acessíveis; racionalizar a produção, uso e trâmite dos itens documentais. Além disso, deverá refletir a organicidade e o inter-relacionamento entre os documentos, bem como a estrutura da instituição que os gerou.

As abordagens até então apresentadas refletem a discussão que se pretendeu realizar sobre a teoria de Hjørland como uma maneira de se compreender as instituições para se entender os documentos arquivísticos por ela produzidos, utilizando-se dessas metodologias para melhorias na gestão de documentos arquivísticos, implementação de sistemas informatizados de gerenciamento de documentos e disseminação da informação em arquivos permanentes. As próximas abordagens apresentadas não se aproximam das rela- ções que se realizou, mas complementam a análise da teoria do autor alemão:

i) Estudos bibliométricos (Bibliometrical studies):

Bibliometria são os estudos realizados, dentro da Ciência da Informação, para levantar características quantitativas da produção, uso e disseminação de informação científica. Apoiado em métodos matemáticos é possível determinar quais são os autores e periódicos mais significativos de um domínio. Hjørland (2002) aponta os estudos bibliométricos como uma ferramenta e um método na análise do domínio que pode ser aplicado de diversas maneiras. Em arquivos a realidade é outra. Essa abordagem não se aplicaria a não ser para estudos da própria disciplina Arquivologia. Quando aplicado a um arquivo específico o método não trará resultados. Numa instituição, por exemplo, a autoria é única, partindo-se do pressuposto que ela possui um único fundo.

j) Estudos epistemológicos e críticos (Epistemological and critical studies):

Hjørland (2002) afirma que todos os tipos de pesquisa são governados por diferentes hipóteses, conhecimento prévio (background), teorias etc. Aborda a importância de definições de paradigmas para as áreas de estudo e a importância do estudo de paradigmas para a análise de domínio. 
Apresenta a epistemologia como sendo mais que uma disciplina de filosofia, mas como uma generalização e interpretação da experiência científica acumulada. $\mathrm{O}$ autor propõe uma classificação das posições epistemológicas: 1) Empirismo/positivismo; 2) Racionalismo; 3) Historicismo/hermenêutica/fenomenologia; 4) Ecletismo, pós-modernismo e ceticismo. Afirma que essa divisão é uma generalização de epistemologias existentes; representam tipos ideais e que não existem isoladamente ("in pure form"). Mais à frente, o autor conclui que os estudos epistemológicos e críticos dos domínios do conhecimento fornecem as bases de conhecimento dos domínios e as avaliações críticas que seu conhecimento reivindica. Fornecem instruções para a seleção, a organização e a recuperação das necessidades de informação e dos critérios de relevância. "Todas as abordagens restantes à análise do domínio se tornam superficiais se os estudos epistemológicos forem negligenciado" (HJøRLAND, 2002, p. 440).

Sabendo que a epistemologia está ligada ao estudo crítico dos princípios, hipóteses e resultados das ciências já constituídas, ou seja, representa a teoria das ciências, esta abordagem está pouco relacionada com a aproximação que se pretende fazer com esse estudo, que é o de identificar a instituição produtora de massas do- cumentais arquivísticas como domínios. Esta abordagem compreende, principalmente as disciplinas consolidadas, ou seja, áreas de conhecimento estudadas e ministradas em um ambiente escolar ou acadêmico, como é a arquivologia, por exemplo.

Uma análise epistemológica da instituição detentora de um arquivo, no entanto, poderia resultar nos objetivos apontados por Hjørland (2002), que são fornecer instruções para a organização, a seleção e a recuperação das necessidades informacionais, mas pouco resultaria para a recuperação e disseminação da informação, que é o maior interesse dos arquivos.

k) Estrutura e instituições da comunicação científica (Structures and institutions in scientific communication):

Esta abordagem está relacionada à comunicação explicitamente científica, à produção de fontes primárias, secundárias e terciárias de informação, ou seja, da produção de documentos internamente por um domínio e suas relações diretas com as fontes informacionais externas de outros domínios. Hjørland (2002, p. 448) conclui que o estudo das estruturas da divisão de trabalho interna dentro dos domínios e da troca de informação entre domínios, fornece a informação útil para a compreensão da função de tipos específicos dos docu- 
mentos e dos serviços de informação e para a construção de guias de literatura.

Esta abordagem é explicitamente ligada a domínios científicos. Qualquer aproximação que se pretenda realizar com instituições que possuem arquivos ficará, no mínimo, forçada. A estrutura de produções de fontes (primárias, secundárias e terciárias) inexiste em arquivos e documentos externos não são considerados, mesmo que façam referência ao fundo em questão, por terem sido produzidos com outros propósitos e em outros conjuntos documentais.

\section{CONSIDERAÇÕES FINAIS}

A partir da análise de cada uma das abordagens propostas por Birger Hjørland, depreende-se que $\mathrm{o}$ entendimento da instituição geradora de um arquivo como um domínio específico trará inúmeros benefícios.

Primeiramente será possível adequar os instrumentos de busca e acesso à informação à realidade da entidade que custodia o arquivo. As tecnologias da informação poderão ser utilizadas como ferramentas que contribuem para o acesso e o uso dos documentos e definindo-se necessidades reais de implantação de sistemas eletrônicos de gerenciamento de documentos arquivísticos como uma necessidade evidente das organizações que produzem uma massa considerável de documentos arquivísticos.

Poder-se-á, ainda, identificar termos mais usuais em detrimento de outros não utilizados na comunidade específica, para que o sistema de recuperação da informação possa ser construído sob uma base sólida de comunicação eficaz, a partir da definição de um vocabulário controlado. Direcionando-se a extração de termos para a realidade específica que circunda 0 documento arquivístico existente na entidade em estudo, a recuperação da informação será modelada de acordo com exigências internas.

As aplicações dos métodos propostos pelo autor alemão poderão solucionar os problemas de concepção de planos de classificação e quadros de arranjo, para que ambos tornem-se instrumentos de representação e recuperação da informação que reflitam a entidade e englobem a complexidade das práticas de produção, recebimento, uso e arquivamento de documentos. $\mathrm{A}$ análise da história administrativa que gerou o fundo, será útil como forma de entender as relações entre os documentos e a configuração dos mesmos como elementos de provas de ações, bem como será fundamental para a obtenção de resultados 
no processamento técnico dos documentos em questão.

Compreender os usuários que produzem, recebem, utilizam e armazenam documentos de acordo com suas necessidades, não de localização dos itens - que é exigência primeira - mas de acesso à informação para a tomada de decisão que é fundamental para as organizações que gerenciam seus documentos arquivísticos.

Definir tipologias documentais, de acordo com o gênero, a espécie e o tipo, de uma maneira que a produção dos documentos seja racionalizada e os processos de busca e acesso possam ser melhores padronizados e também forma de agilizar a elaboração de Tabelas de Temporalidade.

\section{REFERÊNCIAS}

CENTRO DE INFORMAÇÃO DE ACERVOS DOS PRESIDENTES DA REPÚBLICA. Apresentação. Disponível em: $<$ http://www.an.arquivonacional.gov.br/crap p_site/default.asp>. Acesso em: 2 out. 2007.

CONSELHO NACIONAL DE ARQUIVOS CONARQ. e-ARQ Brasil: modelo de requisitos para sistemas informatizados de gestão arquivística de documentos. Rio de Janeiro: CONARQ, 2006. Disponível em: $<$ http://www.conarq.arquivonacional.gov.br/ Media/publicacoes/earqbrasilv1.pdf>. Acesso em: 14 nov. 2006.
EUROPEAN COMMISSION. INTERCHANGE OF DATA BETWEEN ADMINISTRATIONS - IDA. Model Requirements for the Management of Electronic Records - MoReq. Bruxelles: CECA/CEE/CEEA, 2001.

HJØRLAND, B. Domain analysis in information science: eleven approaches - tradional as well as innovative. Journal of Documentation, London, v. 58, n. 4, p. 422462, 2002.

. Domain analysis in information science. In: KENT, A. Encyclopedia of library and information science. New York: Marcel Dekker, 2004. p. 1-7. Disponível em: $<$ www.dekker.com/servlet/product/DOI/101 081EELIS120024990>. Acesso em: 2 out. 2007.

JARDIM, J. M. A Arquivística e as novas tecnologias de informação. Estudos Históricos, Rio de Janeiro, v. 5 , n. 10, p. 251260, 1992.

PAES, M. L. Arquivo: teoria e prática. 3. ed. Rio de Janeiro: FGV, 2005.

SILVA, A. M. et al. Arquivística: teoria e prática de uma ciência da informação. Porto: Afrontamento, 1999.

ZINS, C.; GUTTMANN, D. Domain analysis of social work: an exemaple of an integrated methodological approach. Knowledge Organization, Wurzburg, v. 30, n. 3/4, p. 196-212, 2003.

\section{Title}

An understanding of archival funds under the focus of the domain analysis. 


\begin{abstract}
This paper searches an approximation to Birger Hjørland's theory, identifying public and private institutions that produces archives documents in specific areas of knowledge, which: (a) produce documents related to its activities and functions; (b) follow a specialized terminology; (c) require structural and historical analysis for the determination of organic documents and the establishment of the provenance principle; (d) cover a community of users with complex and specific informational needs, (e) apply intelligent systems for archives documents' retrieval and organization. Based on the proposed approaches it is possible to achieve improvements in the document management process, regarding some aspects, such as: the provenance maintenance and the documents' authenticity, the optimization of record-keeping systems and the archive information retrieval effectiveness.
\end{abstract}

\section{Keywords}

Domain Analysis. Archives Documents Management. Information Organization. Information Retrieval. Archives.

\section{Título}

La comprensión de los fondos archivísticos mediante el enfoque del análisis de campo.

\section{Resumen}

O texto intenta buscar un acercamiento con la teoría de Birger Hjørland, identificando las instituciones públicas y privadas productoras de documentos de archivo como áreas específicas de conocimiento, qué: (a) producem documentos derivados de sus actividades y funciones; (b) tienen terminología especializada; (c) requieren análisis estructurales y históricos para determinar los documentos orgánicos y el establecimiento del principio de procedencia; (d) cubren una comunidad de usuarios con necesidades de acceso a los documentos y necesidades de información compleja y específica; y (e) hacen uso de sistemas inteligentes para la recuperación y organización de documentos de archivo. El uso de los enfoques propuestos podrá lograr mejoras en la gestión de documentos arquivísticos en relación con el mantenimiento de procedencia y autenticidad de los documentos, optimización de los sistemas de gestión de documentos y la eficacia en la recuperación de la información en archivos.

\section{Palabras Clave}

Análisis de Campo. Gestión de Documentos de Archivo. Organización de Información. Recuperación de Información. Archivos.

Recebido em: 09.05.2008

Aceito em: 03.02.2009 